\title{
Aspergillus fumigatus infection in an ostrich with an enlarged neck due to respiratory problems
}

\author{
Gye-Hyeong Woo* \\ Laboratory of Histopathology, Department of Clinical Laboratory Science, Semyung University, Jecheon 27136, Korea
}

\begin{abstract}
This study describes a sudden death in an ostrich (Struthio camelus) with a big neck. Grossly, the air sacs were thickened. Yellow to white, round or coalescent material was scattered on the air sacs. However, the cervical air sac was normal. Histopathologically, multinucleated giant cells, heterophils, and macrophages had infiltrated the air sacs, and many hyphae were seen in the air sacs and on their surfaces. Pyogranulomatous inflammation with intralesional hyphae was observed throughout the lungs. Aspergillus fumigatus was identified microbiologically. In conclusion, the affected animal died because of the respiratory disturbance caused by mycotic airsacculitis and pneumonia.
\end{abstract}

Keywords: Aspergillus fumigatus, hyhae, ostrich, pyogranulomatous airsacculitis, pneumonia

\section{*Corresponding author}

Gye-Hyeong Woo

Laboratory of Histopathology, Department of Clinical Laboratory Science, Semyung University, 65 Semyeong-ro, Jecheon 27136, Korea

Tel: +82-31-649-1539

Fax: +82-31-649-1537

E-mail: ghwoo@naver.com

ORCID:

Gye-Hyeong Woo

https://orcid.org/0000-0002-6947-0241

Conflict of Interest

The author declares no conflicts of interest.

Received: September 18, 2019

Revised: October 30, 2019

Accepted: November 5, 2019
Aspergillosis is an opportunistic infection in animals and humans caused by fungi in the genus Aspergillus. Aspergillus species are distributed widely in the environment, including the soil, air, and decaying vegetation [1-5]. Aspergillus species are an important cause of morbidity and mortality in birds. Avian aspergillosis typically causes respiratory problems [2,4-6]. In the ostrich (Struthio camelus), Aspergillus infections have been reported in the respiratory tract, including the nasal cavity, air sacs, and lungs [7-9], and affected birds often have large necks that have been caused by this condition [10]. However, there are no reports on the pathogenesis of the size of the neck. In this study, we describe granulomatous airsacculitis and pneumonia caused by Aspergillus fumigatus in an ostrich with a big neck and also clarify the reason for the large neck.

A 1-year-old male ostrich from a farm near Seoul, Korea was submitted for a diagnostic investigation. The bird rapidly developed severe clinical signs, such as anorexia, depression, and lethargy, and died suddenly. No clinical problems were observed in any other birds from this farm. The neck of the affected ostrich was markedly enlarged to more than three times its normal size (Fig. 1A and B). The bird was very emaciated. At necropsy, tissues from the brain, lungs, air sacs, heart, liver, spleen, kidneys, trachea, skin, proventriculus, gizzard, and intestine were fixed in $10 \%$ buffered formalin, embedded in paraffin, and sectioned at $4 \mu \mathrm{m}$. The sections were stained with hematoxylin and eosin (H\&E) and periodic acid Schiff (PAS) for histopathology. To examine mycology, direct microscopic examination of the air sacs was performed with Lactophenol cotton blue stain (Fig. 2A). Tissue from the lungs and air sacs was cultured on Sabouraud dextrose agar and incubated at $25^{\circ} \mathrm{C}$ for 3-7 days. A fecal sample was collected for testing, and lung samples were cultured on both blood and MacConkey agars for bacterial isolation.

Direct microscopy examination showed features of Aspergillus species, such as septate hyphae, conidiophores, vesicles, and conidia (Fig. 2B). Fungi grew rapidly in fungal cultures. Initially, the colonies were white, but they became velvet white with a bluish-green center. Microscopically, the conidiophores connected with septate hyphae that were short, smooth, and hyaline. The vesicles expanding from the conidiophores were flask-shaped, 15-22 $\mu \mathrm{m}$ in diameter, and formed a single series of phialides on the upper $2 / 3$ of the conidiophores only. The conidia that developed from the uniseriate phialides 

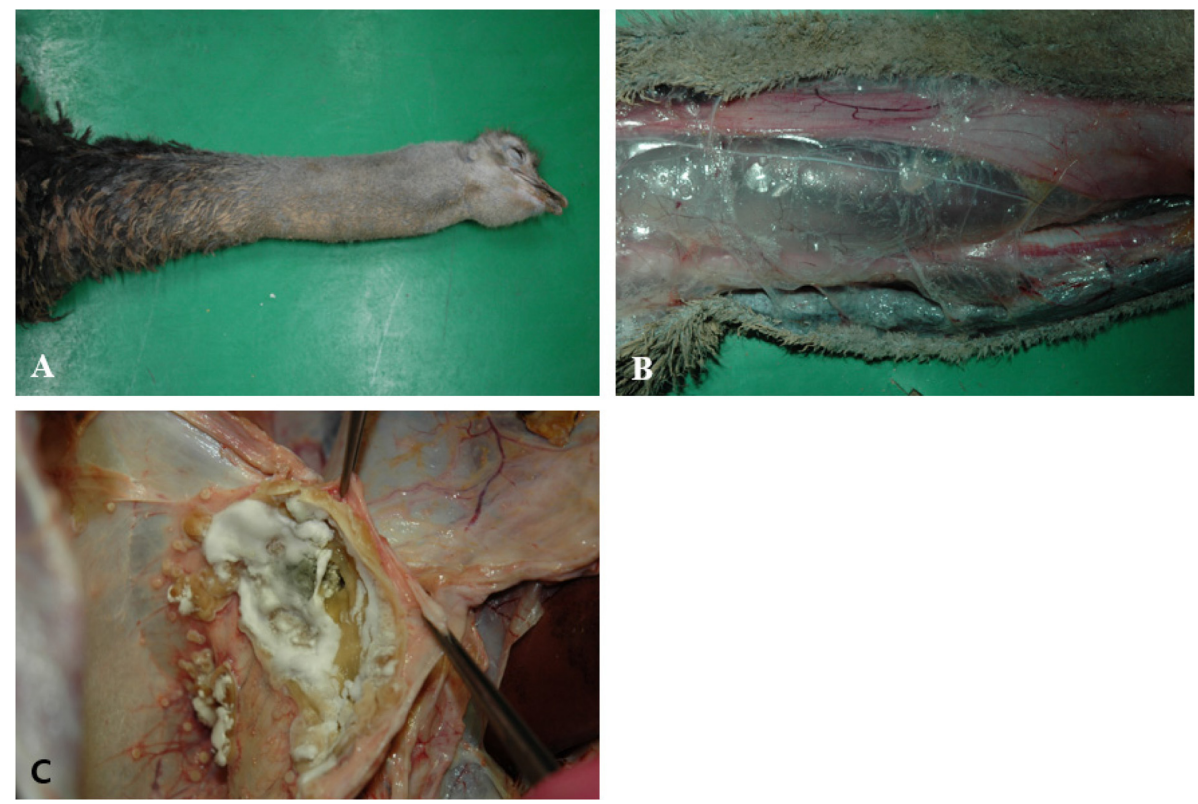

Fig. 1. Gross finding in the ostrich. (A) Enlarged neck. (B) Enlargement of the cervical air sac due to air accumulation. (C) Thickened thoracic air sac with various-sized, yellow, round, crateriform materials.

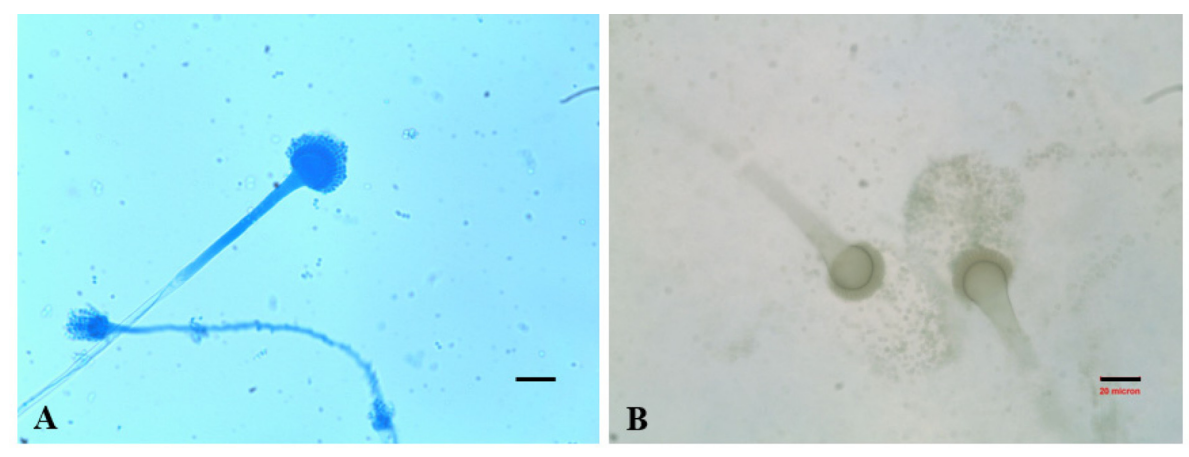

Fig. 2. Direct microscopic examination for mycology. (A) Direct stamp from the thoracic air sac. Conidiophores, vesicles, and conidia are seen. Lactophenol cotton blue stain (Scale bar $=20 \mu \mathrm{m}$ ). (B) Direct stamp from a culture plate. Conidiophores, flask-shaped vesicles, phialides, and chains of conidia are observed (Scale bar $=20 \mu \mathrm{m})$.

were globular to broadly ellipsoidal, $2.1-2.5 \mu \mathrm{m}$ in diameter, and finely rough-walled (Fig. 2). When the conidia were produced, the fungi became green in color. Based on mycology and pathological findings, the organism was identified as $A$. fumigatus. No parasite eggs or bacteria were detected.

Grossly, the walls of the thoracic air sacs were thickened, and various-sized, yellow, round, crateriform material and white to green mold were scattered on the inner surfaces of the thoracic air sacs (Fig. 1C). The inner surfaces of the abdominal air sacs were covered with 4-6-mm, single or coalescent, yellow, round, crateriform material. The surfaces and cut surfaces of the lungs were scattered with 1-5-mm white to yellow foci. The cervical air sacs were simply expanded (Fig. 1B). No other gross lesions were seen.

Histopathologically, multifocal to coalescing pyogranulomatous inflammation was present in the air sacs and lungs
(Fig. 3A and B). The centers of the pyogranulomas consisted of cell debris, including heterophils and numerous hyphae surrounded by multinucleated giant cells and macrophages. The cell debris and hyphae also covered the inner surfaces of the air sacs. Many conidia and conidial heads were observed on the inner surface bordered by air. On PAS staining, pale-red colored positive reactions were seen in the hyphae at the centers of the pyogranulomas (Fig. 3C). No histopathological findings were observed in other organs or tissues.

The diagnosis of Aspergillus infection was based on the morphological characteristics of the fungus and the isolation of $A$. fumigatus from air sacs and lungs. A. fumigatus is the main agent of avian aspergillosis [2,4-6], although Aspergillus flavus has been isolated in some cases [6,11]. These two fungi differ morphologically; A. fumigatus has conidiophores 


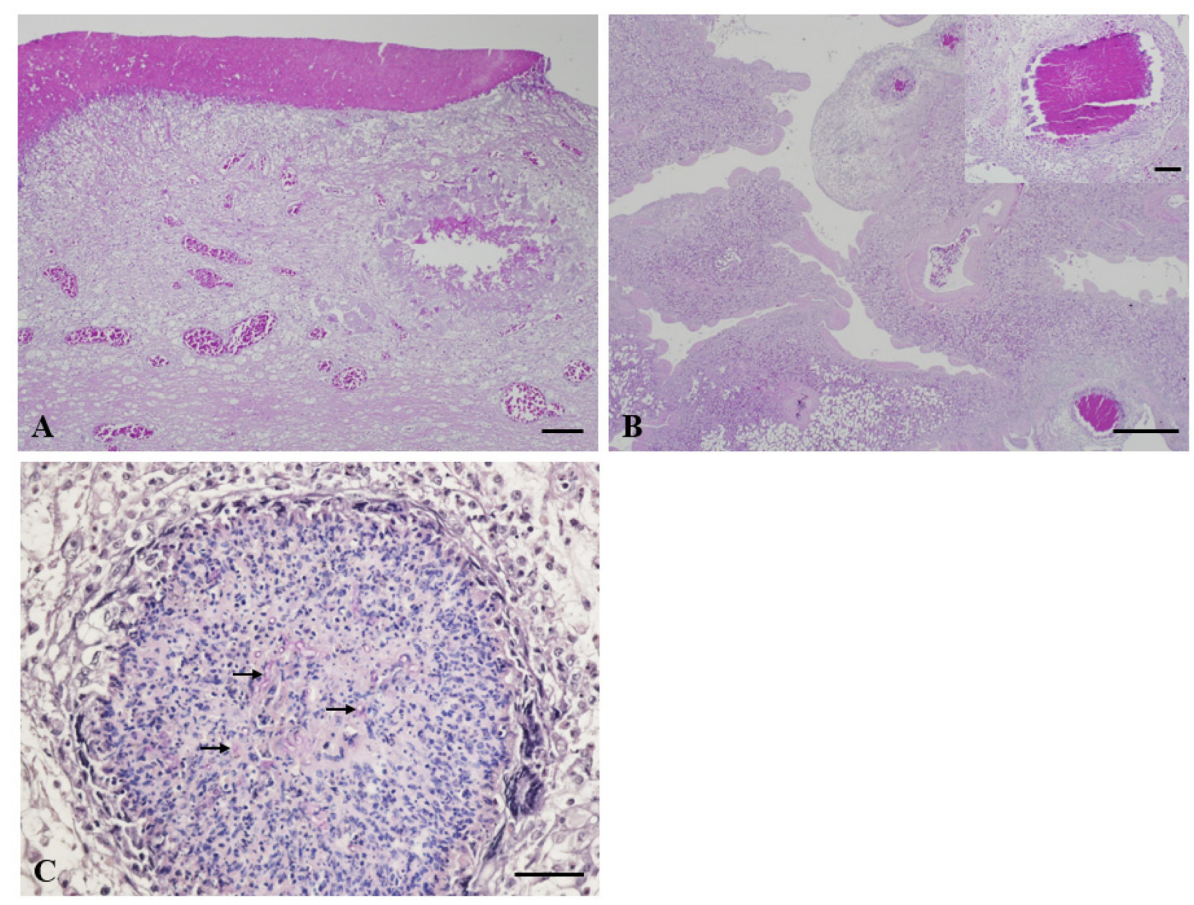

Fig. 3. Histopathology of the thoracic air sac and lungs. (A) A focal granuloma with a central necrotic area in a thickened air sac and necrotic materials attached to the air sac $(\mathrm{H} \& \mathrm{E}$, Scale bar $=50 \mu \mathrm{m})$. (B) Multifocal granulomas with a central necrotic area in the lungs $(H \& E$, Scale bar $=200 \mu \mathrm{m})$. Insert: Magnification of the section shown in Figure 3B (H\&E, Scale bar $=20 \mu \mathrm{m})$. (C) Granulomatous inflammation with intralesional fungus (arrows) (Periodic acid Schiff stain, Scale bar $=20 \mu \mathrm{m}$ ).

with club- or flask-shaped vesicles, phialides, and columnar conidial heads, whereas $A$. flavus has conidiophores with globose vesicles, phialides, and radiate conidia [6]. A. fumigatus, A. flavus, and Aspergillus niger have been identified as causative organisms of ostrich aspergillosis [11,12].

Avian aspergillosis can cause high rates of morbidity and mortality in young animals and, sporadically, in adults [2,6]. Although several lesions are observed in Aspergillus infection, the characteristic lesions of this disease appear primarily in two patterns: airway aspergillosis and systemic aspergillosis $[5,6]$. The airway form is the most common in birds, including chickens, turkeys, captive raptors, and penguins, and is found frequently in young animals. Airway aspergillosis is also the main form in young ostriches [6]. Systemic aspergillosis has been documented in young and mature ostriches $[6,9]$, and the characteristic lesions have been observed in the spleen, gastrointestinal tract, and liver. In our case, multifocal granulomas were restricted to the air sacs and the lungs of a mature bird, which had the airway form of aspergillosis.

Gross edema of the neck has been identified in a previous report [10]. Our case also had a large neck caused by expansion of the cervical air sacs. In birds, the air flows in one direction through the lungs. The air passes through the trachea and moves into the posterior air sacs and lungs, and it then flows into the anterior air sacs through parabronchi, whereas the air flows out of the posterior air sacs through parabronchi and the trachea and simultaneously moves out of anterior air sacs via the trachea. Therefore, in our case, the large neck was induced by the accumulation of air in the cervical air sacs, indicating the failure of air flow, especially during exhalation, because of thickened thoracic air sacs. The ostrich has paired cervical, lateral, and medial clavicular, cranial and caudal thoracic, and abdominal air sacs [13]. In conclusion, the affected bird died because of the respiratory disturbance caused by mycotic airsacculitis and pneumonia.

\section{Acknowledgments}

This study was supported by a Semyung University Research Grant of 2018.

\section{References}

1. Bennett JW. An overview of the genus Aspergillus. In: Machida M, Gomi K (eds). Aspergillus: Molecular Biology and Genomics. pp. 1-17, Caister Academic Press, Norfolk, 2010.

2. Fischer D, Van Waeyenberghe L, Failing K, Martel A, Lierz M. Single tracheal inoculation of Aspergillus fumigatus conidia induced aspergillosis in juvenile falcons (Falco spp.). Avian Pathol 2018;47:33-46.

3. Nemeth NM, Gonzalez-Astudillo V, Oesterle PT, Howerth EW. A 5-year retrospective review of avian diseases diagnosed at the department of pathology, University of Georgia. J Comp Pathol 2016;155:105-120. 
4. Sabino R, Burco J, Valente J, Veríssimo C, Clemons KV, Stevens DA, Tell LA. Molecular identification of clinical and environmental avian Aspergillus isolates. Arch Microbiol 2019;201:253-257.

5. Seyedmousavi S, Guillot J, Arn P, de Hoog GS, Mouton JW, Melchers WJ, Verweij PE. Aspergillus and aspergilloses in wild and domestic animals: a global health concern with parallels to human disease. Med Mycol 2015;53:765-797.

6. Charlton BR, Chin RP, Barnes HJ. Fungal infections. In: Swayne DE, Glisson JR, McDougald LR, Nolan LK, Suarez DL, Nair VL (eds.). Diseases of Poultry. 13th ed. pp. 10771096, John Wiley \& Sons Inc., Ames, 2013.

7. Fitzgerald SD, Moisan PG. Mycotic rhinitis in an ostrich. Avian Dis 1995;39:194-196.

8. Khosravi AR, Shokri H, Ziglari T, Naeini AR, Mousavi Z, Hashemi H. Outbreak of severe disseminated aspergillosis in a flock of ostrich (Struthio camelus). Mycoses 2008;51:557559.

9. Yokota T, Shibahara T, Wada Y, Hiraki R, Ishikawa Y, Kadota K. Aspergillus fumigatus infection in an ostrich (Struthio camelus). J Vet Med Sci 2004;66:201-204.

10. Rousseaux CG, Dalziel JB. Aspergillus pneumonia in an ostrich (Struthio camelus). Aust Vet J 1981;57:151-152.

11. Perelman B, Kuttin ES. Aspergillosis in ostriches. Avian Pathol 1992;21:159-163.

12. Pérez J, García PM, Méndez A, Astorga R, Luque I, Tarradas C. Outbreak of aspergillosis in a flock of adult ostriches (Struthio camelus). Vet Rec 2003;153:124-125.

13. Bezuidenhout AJ, Groenewald HB, Soley JT. An anatomical study of the respiratory air sacs in ostriches. Onderstepoort J Vet Res 1999;66:317-325. 